\title{
NÍVEIS DE VITAMINA D SOBRE O DESEMPENHO E DESENVOLVIMENTO ÓSSEO DE LINHAGENS DE FRANGOS DE CORTE
}

\author{
LEVELS AND SOURCES OF VITAMIN D ON PERFORMANCE AND BONE DEVELOPMENT OF \\ STRAINS IN BROILERS
}

\author{
R. P. OLIVEIRA ${ }^{1}$, E. T. SANTOS ${ }^{2}$, S. SGAVIOLI ${ }^{1}$, R. G. GARCIA ${ }^{1}$, \\ S. M. BARALDI-ARTONI ${ }^{2}$, D. E FARIA ${ }^{3}$
}

\section{RESUMO}

A disponibilidade e o nível adequado de vitamina $\mathrm{D}$ deve atender à demanda físiológica para formação óssea, e reduzem a ocorrência de problemas locomotores em frangos de corte. Com o objetivo de avaliar três níveis de vitamina D sobre o desempenho, força de ruptura e conteúdo mineral das tíbias de três linhagens de frangos de corte, foram utilizado 1.440 pintos de um dia, distribuídos em um delineamento inteiramente casualizado em esquema fatorial 3x3, com três linhagens (Ross 308, Cobb 500 e Hybro) e três níveis de inclusão de vitamina $\mathrm{D}_{3}\left(1250\right.$ UI vitamina $\mathrm{D}_{3} ; 3000$ UI vitamina $\mathrm{D}_{3}$ e 2760 UI de hidroxicolecalciferol $25(\mathrm{OH}) \mathrm{D}_{3}$, fornecidos na ração até o $21^{\circ}$ dia de vida, com oito repetições de 30 aves por parcela, totalizando 160 aves por tratamento. Não houve diferenças $(\mathrm{P}>0,05)$ entre os níveis de vitamina $\mathrm{D}$, e as linhagens para o desempenho e força de ruptura, exceto para o consumo de ração para o fator linhagem $(\mathrm{P}<0,05)$, devido ao seu potencial de crescimento. Os níveis de vitamina $\mathrm{D}$ não influenciaram $(\mathrm{P}>0,05)$ as quantidades de cálcio e fósforo nas tíbias. No entanto, para as linhagens houve diferença significativa $(\mathrm{P}<0,05)$ aos 14 dias de idade, com maior quantidade de cálcio para o Ross 308 e maior quantidade de fósforo para o Hybro, evidenciando diferenças no metabolismo do cálcio e do fósforo em frangos de corte. Em conclusão a adição de 1250 UI de vitamina $\mathrm{D} / \mathrm{kg}$ de ração é suficiente para garantir o desempenho e o desenvolvimento ósseo de frangos de corte, independente da linhagem a ser utilizada, mediante ao ajuste correto dos níveis de Ca e P.

PALAVRAS-CHAVE: Cálcio. Força de ruptura. Fósforo. Hidroxicolicalciferol. Linhagens.

\section{SUMMARY}

The availability and appropriate level of vitamin D to promote physiological demand, bone formation and reduce the occurrence of locomotors problems in broilers. The aim of this study was to evaluate three levels of vitamin D on performance breaking strength and mineral content of tibia of three strains of broilers, it was used 1,440 chicken of one day of age. The complete random design used was in factorial 3X3, with three strains (Ross 308, Cobb 500 and Hybro) and three inclusion levels of vitamin D3 (1250 IU vitamin D3; 3000 IU vitamin D3 and 2760 UI of 25hydroxycholecalciferol (OH) D3), provided in the diet until 21 day of life, with eight replicates of 30 birds per group, a total of 160 birds per treatment. There was no difference $(\mathrm{P}>0.05)$ between the levels of vitamin $\mathrm{D}$, in the strains for the perfomance and breaking strength, except for feed intake for the strain factor $(\mathrm{P}<0.05)$, with higher feed intake of Cobb, probably due to its higher growth potential. The levels of vitamin $D$ had no influence significant $(P>0.05)$ the quantities of calcium and phosphorus in the tibia bones. However, for strains was significant difference $(P<0.05)$ at 14 days of age, with higher amounts of calcium to Ross 308 and higher amount of phosphorus to Hybro. This can be explained because of the genetic variation in metabolism of calcium and phosphorus in broilers. In conclusion the addition of $1250 \mathrm{IU}$ vitamin $\mathrm{D} / \mathrm{kg}$ of feed is sufficient to ensure performance and bone development of broilers independent of the strain to be used by proper adjustment of the levels of $\mathrm{Ca}$ and $\mathrm{P}$.

KEY-WORDS: Breaking strength. Calcium. Phosphorus. Strains. 25-hidroxicholecalciferol.

\footnotetext{
${ }^{1}$ Universidade Federal de Grande Dourados, Dourados, MS

${ }^{2}$ Universidade Estadual Paulista Júlio de Mesquita Filho, Jaboticabal, SP

${ }^{3}$ Universidade do Estado de São Paulo, Pirassununga, SP
} 


\section{INTRODUÇ̃̃O}

O frango de corte moderno possui excelente potencial genético, que quando aliado a manejo nutricional e sanitário adequados permite elevado ganho de peso. No entanto, o desenvolvimento do tecido ósseo não acompanha o aumento no ganho de massa muscular, que pode gerar problemas locomotores e outras desordens metabólicas. Estes problemas são preocupantes para a indústria avícola devido os altos índices de descarte nos abatedouros, ocasionados por carcaças mal desenvolvidas e por perdas relativas ao desempenho das aves (KNOWLES et al., 2008, NÄ̈̈S, 2008).

Segundo Coto et al. (2008) as perdas estimadas decorrentes de problemas locomotores em frangos de corte nos EUA, são de 80 milhões de dólares por ano. O maior impacto destas perdas ocorre no abatedouro por condenações de carcaças com processos inflamatórios nas articulações, dermatites e escoriações, provocadas pelo excesso de decúbito das aves que apresentaram dificuldade locomotora e por isso durante a criação são por vezes pisoteadas pelas aves saudáveis (OVIEDO-RONDÓN, 2008).

$\mathrm{O}$ manejo nutricional pode ser uma ferramenta decisiva na diminuição dessas anormalidades ósseas. Estudos apontam que o meio nutricional mais eficaz para se combater problemas ósseos envolve a suplementação dietética com vitamina D (PIZAURO et al., 2002; LEDWABA, 2003). Em estudos realizados se observou que metabolitos da vitamina $D_{3}$ mostraram-se eficazes em relação à discondroplasia tibial (RENNIE \& WHITEHEAD, 1996).

A forma ativa da vitamina $\mathrm{D}$, a 1,25diidroxivitamina $\mathrm{D}_{3} \quad 1,25(\mathrm{OH})_{2} \mathrm{D}_{3}$ é um importante regulador do desenvolvimento do tecido ósseo, do metabolismo e da homeostase do cálcio, possuindo papel fundamental na regulação do crescimento e diferenciação celular do tecido ósseo (BRITO et al., 2010). A $25(\mathrm{OH}) \mathrm{D}_{3}$ tem atividade duas vezes melhor que a vitamina $\mathrm{D}_{3}$ (colecalciferol), além de ser facilmente absorvida pelas células do intestino, quando comparado com o colecalciferol (TORRES et al., 2009).

De acordo com o NRC (1994) a exigência de vitamina $\mathrm{D}_{3}$ para frangos de corte é de $5 \mathrm{mg} / \mathrm{kg}$ (200 $\mathrm{UI} / \mathrm{kg}$ ), Kasim \& Edwards (2000) sugerem um aporte mais elevado da vitamina, e estimaram a exigência em cerca de $25 \mathrm{mg} / \mathrm{kg}$.

Vale ressaltar que a exigência de vitamina $D_{3}$ está relacionada com a quantidade de $\mathrm{Ca}$ e $\mathrm{P}$ disponíveis na dieta. Waldroup et al. (1965) mostraram que o requisito pode variar de $5 \mathrm{mg} / \mathrm{kg}$ sob teores de $\mathrm{Ca}$ e $\mathrm{P}$ normais e, mais de $40 \mathrm{mg} / \mathrm{kg}$ quando as quantidades ou proporções de $\mathrm{Ca}$ e $\mathrm{P}$ foram abaixo do ideal. Williams et al. (2000) e Bar et al. (2003) sugeriram que as necessidades de cálcio para genótipos modernos de frango de corte está próximo de $13 \mathrm{~g} / \mathrm{kg}$ do que o valor de $10 \mathrm{~g} / \mathrm{kg}$ sugerido pelo NRC (1994).

Alguns autores já estudaram a interação entre linhagens de frango de corte e níveis de vitamina $\mathrm{D}_{3}$ (VELTEMANN \& JENSEN, 1981; EDWARDS, 2000;
SHAFEY et al., 1990; ELLIOT \& EDWARDS, 1994), porém nos estudos realizados os autores não descrevem as linhagens utilizadas, ou estas não são utilizadas com frequência no Brasil, portanto, desconhecem-se os efeitos da hidroxicolecalciferol $25(\mathrm{OH}) \mathrm{D}_{3}$ sobre as linhagens utilizadas atualmente no Brasil como Ross 308, Cobb 500 e Hybro.

Mediante informações se torna necessário pesquisas que relacionem níveis de vitamina $\mathrm{D}_{3}$, com o desempenho e desenvolvimento ósseo em frangos de corte. A proposta do estudo foi avaliar o desempenho e o desenvolvimento das tíbias de frangos de corte de diferentes linhagens, alimentados com dietas contendo diferentes níveis de vitamina $\mathrm{D}_{3}$, até os 21 dias de idade.

\section{MATERIAL E MÉTODOS}

\section{Aves e manejo}

Foram utilizados 1.440 pintos de um dia de idade, machos, das linhagens Ross 308, Cobb 500 e Hybro, em um galpão constituído em 72 parcelas (boxes), resultando em 30 aves por parcela, cujos pesos médios foram de $47 \mathrm{~g}, 46 \mathrm{~g}$, e de $48 \mathrm{~g}$ respectivamente, provenientes de matrizes com 60 a 62 semanas de idade. As aves foram criadas em galpão experimental, com programa de iluminação continua e fornecimento de ração e água à vontade, até os 21 dias de idade. Aos sete dias de idades todas as aves foram vacinadas contra as doenças Newcastle, Bronquite aviária e Gumboro (cepa fraca), aos 14 dias de idade as aves foram vacinadas contra Gumboro (cepa forte) via água de bebida, sendo utilizado leite em pó como veículo (2 $\left.\mathrm{g} \mathrm{L}^{-1}\right)$.

Durante todo o período experimental foi mantido o conforto térmico das aves através do manejo de cortinas e campânulas. A cama utilizada foi do tipo maravalha, com 1,2 kg do material por box.

\section{Delineamento e tratamentos experimentais}

$\mathrm{O}$ delineamento experimental utilizado foi $\mathrm{o}$ inteiramente casualizado em esquema fatorial $3 \times 3$, com três linhagens (Ross 308, Cobb 500 e Hybro) e três níveis de inclusão de vitamina $\mathrm{D}_{3}$ (1250 UI vitamina $\mathrm{D}_{3} ; 3000$ UI vitamina $\mathrm{D}_{3}$ e 2760 UI de $\mathrm{HyD} 25(\mathrm{OH}) \mathrm{D}_{3}$, com oito repetições de 30 aves por parcela, totalizando 160 aves por tratamento.

\section{Dietas Experimentais}

63 As dietas foram formuladas a base de milho e le soja, de acordo com as exigências nutricionais para a fase pré-inicial (1 a 7 dias) e inicial (8 a 21 dias), de acordo com as recomendações de Rostagno et al. (2005), exceto para o cálcio e o fósforo, 0,80 e $0,50 \%$, respectivamente, de acordo com as recomendações de Edwards \& Veltman (1983) e Edwards (2000) (Tabela 1). Na ração experimental foi calculada uma porção variável de $0,10 \%$, para inclusão dos tratamentos. A quantidade de vitamina $\mathrm{D}$ dos ingredientes da ração não foi considerada, sendo a mesma para todos os tratamentos. 
Tabela 1 - Composição nutricional e calculada das dietas experimentais nas fases de 1 a 7 e de 8 a 21 dias de idade.

\begin{tabular}{|c|c|c|}
\hline Ingredientes & 1 a 7 dias & 8 a 21 dias \\
\hline Milho & 60,80 & 61,50 \\
\hline Farelo de soja $(45 \%)$ & 34,59 & 33,06 \\
\hline Calcário & 0,43 & 0,43 \\
\hline Fosfato bicálcico & 2,03 & 2,04 \\
\hline Sal & 0,50 & 0,50 \\
\hline Óleo vegetal & 0,36 & 1,56 \\
\hline L-Lisina HCL & 0,43 & 0,26 \\
\hline DL-Metionina & 0,37 & 0,26 \\
\hline L-Treonina & 0,19 & 0,09 \\
\hline Supl.vit. e mineral ${ }^{1}$ & 0,20 & 0,20 \\
\hline Porção variável $^{2}$ & 0,10 & 0,10 \\
\hline Total & 100,00 & 100,00 \\
\hline Energ. Metab.(kcal/kg) & 2.960 & 3.050 \\
\hline Proteína bruta (\%) & 22,11 & 21,14 \\
\hline Cálcio (\%) & 0,80 & 0,80 \\
\hline Fósforo disponível & 0,50 & 0,50 \\
\hline Sódio & 0,22 & 0,22 \\
\hline Met+cis digestível & 0,96 & 0,84 \\
\hline Metionina digestível & 0,66 & 0,55 \\
\hline Lisina digestível & 1,36 & 1,19 \\
\hline Treonina digestível & 0,88 & 0,77 \\
\hline Triptofano digestível & 0,23 & 0,22 \\
\hline
\end{tabular}

${ }^{1}$ Suplemento vitamínico e mineral: quantidade por kg de ração: Vit. A- 9000 UI; Vit. D3- 1250 UI; Vit. E- 20 mg; Vit. K3- 2,5 mg; Vit. B1- 1,5 mg; Vit. B2- 6 mg; Vit. B6- 3 mg; Vit. B12- 12 mcg; Niacina- 25 mg; Acido Pantatênico- 12 mg; Biotina- 60 mcg; Ácido Fólico- 0,8 mg; Selênio- 0,25 mg; Ferro- $50 \mathrm{mg}$; Cobre- $10 \mathrm{mg}$; Zinco- $50 \mathrm{mg}$; Manganês- $80 \mathrm{mg}$; Iodo- $1 \mathrm{mg}$; Cobalto- 1mg; Antioxidante (BHT)- $120 \mathrm{mg} ;{ }^{2}$ Porção variável constituiu de $0,10 \%$ de caulim e/ou vitamina $\mathrm{D}_{3}$, proporcionando $2760 \mathrm{UI}$ de $25(\mathrm{OH})\left(\mathrm{D}_{3}\right) / \mathrm{kg}$ de ração e/ou 1750 UI de colecalciferol $\left(\mathrm{D}_{3}\right) / \mathrm{kg}$ de ração.

\section{Características avaliadas}

\section{Desempenho}

Ao final do período experimental (21 dias de idade) realizou-se a pesagem das aves e sobras de ração para o calculo do ganho de peso, consumo de ração e conversão alimentar.

\section{Força de ruptura}

Foi avaliada a força de ruptura da tíbia esquerda de oito aves por repetição aos 21 dias de idade, por ensaio mecânico (flexão em três pontos e compressão axial) em máquina universal EMIC ${ }^{\circledR}$, modelo DL 3000. Os ossos foram apoiados em dois suportes (dois pontos), de maneira horizontal, sendo a distância de vão de apoio ajustada conforme o tamanho do menor osso. A força foi aplicada no osso em um terceiro ponto, no meio geométrico entre os dois apoios (terço médio do osso). $\mathrm{O}$ ensaio foi destrutivo $\mathrm{e}$ registrou a força de ruptura. A carga de $1000 \mathrm{~N}$ foi aplicada à velocidade de $54 \mathrm{~mm} / \mathrm{min}$, sendo os resultados expressos em $\mathrm{kgf} / \mathrm{cm}^{2}$ (LOUZADA, 1997).

\section{Conteúdo mineral}

Após a realização da análise de força de ruptura as tíbias foram utilizadas nas análises de cálcio e fósforo. As análises dos minerais das amostras dos diferentes tratamentos foram realizadas no Laboratório de Nutrição Animal, da FCAV/UNESP, Campus de Jaboticabal/SP, de acordo com as metodologias descritas por Silva \& Queiroz (2002), sendo expressos em \% em relação à matéria seca desengordurada.

\section{Análise Estatística}

Os dados foram submetidos à análise de variância pelo procedimento General Linear Model (GLM) do programa SAS ${ }^{\circledR}$ (SAS Institute, 2002), em caso de efeito significativo a uma probabilidade de $5 \%$ foi realizado o teste de Tukey.

\section{RESULTADOS E DISCUSSÃO}

Não houve interação significativa $(\mathrm{P}>0,05)$ entre os fatores analisados, linhagem e níveis de vitamina $\mathrm{D}_{3}$ sobre as características de ganho de peso e conversão alimentar de 1 a 21 dias de idade (Tabela 2). O consumo de ração (Tabela 2) foi significativo $(\mathrm{P}<0,05)$ para as linhagens, com maior consumo de ração para a linhagem Cobb 500. Moreira et al. (2004) avaliaram os índices zootécnicos das linhagens Ross, Cobb e Hybro, e verificaram que o Cobb apresentou maior consumo de ração em relação as demais linhagens, semelhante aos resultados obtidos no estudo. Estes resultados se devem ao potencial genético do Cobb linhagens estudadas, apesar do maior consumo de ração para o Cobb, não houve diferença significativa $(\mathrm{P}>0,05)$ para a conversão alimentar entre as linhagens, pois apesar do ganho de peso não ter sido significativo $(\mathrm{P}=0,09)$, este foi maior para o Cobb, não afetando a conversão alimentar. 
Tabela 2 - Valores médios para consumo de ração $(\mathrm{g})$, ganho de peso (g), conversão alimentar e resistência óssea.

\begin{tabular}{lcccc}
\hline $\begin{array}{l}\text { Tratamentos/ } \\
\text { Parâmetros }\end{array}$ & $\begin{array}{c}\text { Consumo de ração } \\
(\mathbf{g})\end{array}$ & $\begin{array}{c}\text { Ganho de peso } \\
(\mathbf{g})\end{array}$ & $\begin{array}{c}\text { Conversão } \\
\text { alimentar }\end{array}$ & Força de ruptura $\left(\mathbf{k g f}_{\mathbf{c}} \mathbf{\mathbf { c m } ^ { 2 } )}\right.$ \\
\hline Ross 308 & $849 \mathrm{~b}$ & 659 & 1,284 & 2,52 \\
Cobb 500 & $893 \mathrm{a}$ & 688 & 1,299 & 2,65 \\
Hybro & $844 \mathrm{~b}$ & 663 & 1,273 & 2,68 \\
\hline 1250 vit. D & 854 & 665 & 1,286 & 3,03 \\
1250 vit. D + HyD & 875 & 681 & 1,288 & 3,03 \\
3000 vit. D + HyD & 857 & 664 & 1,282 & 0,13 \\
\hline Probabilidades & & & & 0,99 \\
Linhagem (L) & 0,01 & 0,09 & 0,74 & 0,43 \\
Vitamina (V) & 0,36 & 0,40 & 0,98 & 19,64 \\
L x V & 0,27 & 0,34 & 0,29 & 6,03 \\
CV (\%) & 5,11 & 4,79 & & \\
\hline CV
\end{tabular}

CV: coeficiente de variação. a-b: médias seguidas por letras distintas nas colunas diferem significativamente pelo teste de Tukey a $5 \%$ de probabilidade. HyD: hidroxicolicalciferol.

Tabela 3 - Valores médios de cálcio e fósforo nos ossos, na matéria seca desengordurada.

\begin{tabular}{lcccccc}
\hline Tratamentos/ & \multicolumn{3}{c}{ Ca $\mathbf{( m g / k g )}$} & \multicolumn{3}{c}{$\mathbf{P}(\mathbf{m g} / \mathbf{k g})$} \\
\cline { 2 - 7 } Parâmetros & $\mathbf{7 ~ d i a s}$ & $\mathbf{1 4}$ dias & $\mathbf{2 1 ~ d i a s}$ & $\mathbf{7 ~ d i a s}$ & $\mathbf{1 4}$ dias & $\mathbf{2 1 ~ d i a s}$ \\
\hline Ross 308 & 7,71 & $7,39 \mathrm{a}$ & 10,11 & 4,91 & $2,66 \mathrm{~b}$ & 5,52 \\
Cobb 500 & 6,68 & $4,83 \mathrm{c}$ & 10,82 & 4,35 & $2,88 \mathrm{~b}$ & 5,77 \\
Hybro & 6,62 & $5,58 \mathrm{~b}$ & 11,73 & 4,69 & $3,34 \mathrm{a}$ & 6,22 \\
\hline 1250 vit. D & 6,79 & 4,85 & 10,32 & 4,70 & 2,79 & 5,79 \\
1250 vit. D + HyD & 7,39 & 5,16 & 11,21 & 4,69 & 2,94 & 5,57 \\
3000 vit. D + HyD & 6,84 & 5,22 & 11,14 & 4,56 & 3,14 & 6,16 \\
\hline Probabilidades & & & & & \\
Linhagem (L) & 0,05 & 0,02 & 0,12 & 0,07 & 0,01 & 0,18 \\
Vitamina (V) & 0,35 & 0,33 & 0,41 & 0,77 & 0,21 & 0,28 \\
L x V & 0,20 & 0,43 & 0,48 & 0,10 & 0,40 & 0,48 \\
CV (\%) & 5,61 & 7,37 & 8,19 & 6,49 & 5,39 & 9,29 \\
\hline
\end{tabular}

CV: coeficiente de variação. a-b: médias seguidas por letras distintas nas colunas diferem significativamente pelo teste de Tukey a $5 \%$ de probabilidade. HyD: hidroxicolicalciferol.

Não houve efeito significativo $(\mathrm{P}>0,05)$ para as características de desempenho frente aos tratamentos com vitamina $\mathrm{D}_{3}$. Como os níveis de cálcio $(\mathrm{Ca})$ e fósforo $(\mathrm{P})$ da dieta foram adequados, a vitamina $\mathrm{D}$ não afetou a deposição tecidual. A interferência no ganho de peso e no rendimento de carcaça poderia acontecer se os níveis de $\mathrm{Ca}$ e $\mathrm{P}$ estivessem desbalanceados, pois, nesta condição, a vitamina D compensaria este desequilíbrio, aumentando a absorção intestinal e diminuindo a excreção renal, para garantir a homeostase destes minerais no organismo (WALDENTEST, 2006).

Entretanto, Whitehead et al. (2004) ao analisarem níveis de vitamina D, de 200, 800, 5000 e 10000 UI em tratamentos com: $0,80 \%$ de $\mathrm{Ca}$ e 0,35 e $0,50 \%$ de $\mathrm{P} ; 1,30 \%$ de Ca e 0,35 e $0,50 \%$ de $\mathrm{P}$ até os 14 dias para frangos de corte, observaram aumento significativo no peso corporal e da resistência óssea, proporcionalmente a adição da vitamina $\mathrm{D}$, independente do nível de Ca e P. Foi demonstrado por Khan et al. (2010), ao estudarem menores níveis de vitamina D $(200,1500,2500$ e 3500 UI de colecalciferol) que os melhores resultados para peso corporal, conversão alimentar aos 28 e 42 dias, foram obtidos para o nível máximo utilizado de vitamina $\mathrm{D}$ utilizado, de 3500 UI.
Não houve efeito significativo $(\mathrm{P}>0,05)$ das diferentes níveis de vitamina $\mathrm{D}$ para a força de ruptura das tíbias dos frangos de corte nas diferentes idades (7, 14 e 21 dias de idade). Semelhantes aos resultados Garcia et al. (2013) não encontraram diferença significativa para a resistência óssea, fornecendo colecalciferol $\left(\mathrm{D}_{3}\right)$, e seus metabólitos $1,25(\mathrm{OH})_{2} \mathrm{D}_{3}$, $25(\mathrm{OH}) \mathrm{D}_{3}$ e $1 \alpha(\mathrm{OH}) \mathrm{D}_{3}$. Segundo Aburto et al. (1998), os efeitos da vitamina $\mathrm{D}_{3}$ e dos metabólitos podem variar dependendo da resposta biológica, devido aos fatores externos, como os ambientais e internos como a variação genética. Portanto, de acordo com os dados de força de ruptura, em dietas com níveis adequados de Ca e P, a inclusão de 1250 UI de vitamina D/kg de ração, é suficiente para o desenvolvimento das tíbias das aves, mantendo a força de ruptura.

A absorção de cálcio no intestino pode ser aumentada utilizando-se os metabólitos da vitamina D. Isso ocorre devido à redução da formação de complexos de cálcio e, portanto, pode contribuir com o desenvolvimento ósseo das aves (APPLEGATE et al., 2003;. MILLER et al., 2006). No entanto, não houve aumento na quantidade de cálcio das tíbias das aves alimentadas com $25(\mathrm{OH})_{2} \mathrm{D}_{3} \quad(\mathrm{P}>0,05)$. Porém, nas linhagens aos 14 dias de idade (Tabela 4), a quantidade de cálcio e fósforo nas tíbias foi significativa $(\mathrm{P}<0,05)$. 
A linhagem Ross 308 foi a que apresentou a maior quantidade de cálcio $(7,39 \mathrm{mg} / \mathrm{kg})$ aos 14 dias de idade, em comparação as demais linhagens. Para o fósforo a linhagem que apresentou a maior quantidade $(3,34$ $\mathrm{mg} / \mathrm{kg}$ ) do mineral nas tíbias foi a Hybro (Tabela 3). Isso pode ser explicado devido à variação genética no metabolismo do cálcio e do fósforo em frangos de corte (BECKER et al., 1991).

SHAFEY et al., 1990 verificaram a interação entre a suplementação de vitamina $\mathrm{D}$ (4 e $8 \mathrm{mg}$ de colecalciferol $/ \mathrm{kg}$ de ração) e linhagens de frango de corte comercial, em que ocorreu aumento significativo $(\mathrm{P}<0,05)$ de $\mathrm{Ca}$ e $\mathrm{P}$ nas tíbias, no entanto, os autores não revelaram as linhagens utilizadas no estudo.

\section{CONCLUSÕES}

A adição de 1250 UI de vitamina $\mathrm{D} / \mathrm{kg}$ de ração é suficiente para garantir o desempenho e o desenvolvimento ósseo de frangos de corte, independente da linhagem a ser utilizada, mediante ao ajuste correto dos níveis de $\mathrm{Ca}$ e $\mathrm{P}$.

\section{REFERÊNCIAS}

APPLEGATE, T. J.; ANGEL, R.; ClASSEN, H. L. Effect of dietary calcium, 25-hydroxycholecalciferol, and bird strain on small intestinal phytase activity in broiler chickens. Poultry Science, v.82, p.1140-1148, 2003.

BAR, A.; SHINDER, D.; YOSEFI, S.; VAX, E.; PLAVNIK, I. Metabolism and requirements for calcium and phosphorus in the fast-growing chicken as affected by age. British Journal of Nutrition, v.89, p.51-60, 2003.

BRITO, J. A. G.; BERTECHINI, A. G.; FASSANI, E. J.; RODRIGUES P. B.; LIMA, E. M. C.; MENEGHETTI, C. Efeito da vitamina D3 e 25-hidroxicolecalciferol sobre o desempenho, o rendimento de carcaça e a morfologia intestinal de frangos de corte. Revista Brasileira de Zootecnia, v.39, n.12, p.26562663, 2010.

COTO, Z.; YAN, F.; CERRATE, S.; WANG, Z.; SACAKLI, P.; HALLEY, J.; WIERNUSZ, C.; MARTINEZ, A.; WALDROUP, P. Effects of Dietary Levels of Calcium and Nonphytate Phosphorus in Broiler Starter Diets on Live Performance, Bone Development and Growth Plate Conditions in Male Chicks Fed a Corn-Based Diet. International Journal of Poultry Science, v.7, n.7, p.638-645, 2008.

EDWARDS, H. M. Nutrition and skeletal problems in poultry. Poultry Science, v.79, p. 1018-1023, 2000. EDWARDS, H. M.; VELTMAN, J. R. The role of calcium and phosphorus in the etiology of tibial dyschondroplasia in young chicks. Journal of. Nutrition, v.113, p.1565-1575, 1983.

ELLIOT, M. A; EDWARDS, H. M JR. Effect of Genetic Strain, Calcium, and Feed Withdrawal on
Growth, Tibial Dyschondroplasia, Plasma 1,25Dihydroxycholecalciferol, and Plasma 25Hydroxycholecalciferol in Sixteen-Day-Old Chickens. Poultry Science, v.73, p.509-519, 1994.

GARCIA, A. F. Q. M.; MURAKAMI, A. E.; AMARAL DUARTE, C. R.; OSPINA ROJAS, I. C.; PICOLI, K. P.; PUZOTTI, M. M. Use of Vitamin $\mathrm{D}_{3}$ and Its Metabolites in Broiler Chicken Feed on Performance, Bone Parameters and Meat Quality. Asian-Australasian Journal of Animal Sciences (Print), v.26, p.408-415, 2013.

KASIM, A.; EDWARDS, H. M., JR. Evaluation of cholecalciferol sources using broiler chick bioassays. Poultry Science, v.79, p.1617-1622, 2000.

KHAN, S. H.; SHAHID, R.; MIAN, A. A.; SARDAR, R.; ANJUM, M. A. Effect of the level of cholecalciferol supplementation of broiler diets on the performance and tibial dyschondroplasia. Journal of Animal Physiology and Animal Nutrition, v.94, n.5, 584-593, 2010.

KNOWLES, T. G. Leg disorders in broiler chickens: prevalence, risk factors and prevention. Journal of Bone, p. 1545, 2008.

LEDWABA, F. M.; ROBERSON, K. D. Effectiveness of 25- hidroxycholecalciferol in the prevention of tibial dyschondroplasia in Ross cockerels depends on dietary calcium level. Poultry Science, v.82, p.1769-1777, 2003.

LOUZADA, M. J. Q.; PELÁ, C. A.; BELANGERO, W. D.; SANTOS-PINTO, R. Densidade de peças ósseas de frangos. Estudo pela densitometria óptica radiográfica. Veterinária e Zootecnia, São Paulo, v.9, p.95-109, 1997.

MILLER, E. H. Vitamin D insufficiency in male osteoporosis. Clinic Cornerstone, v. 8, p. 14-19, 2006. NÄÄAS, I. A. Deficiências locomotoras em frangos de corte e bem-estar animal. Revista Brasileira de Ciência Avícola, n. 2, 2008.

NRC, Nutrient Requirements of Poultry, 9th rev. edn (Washington, DC, National Academy Press) 1994. OVIEDO-RONDÓN, E. O. Leg Health in Large Broilers. NC Broiler Supervisors' Short Course Poultry Science, v.55, p.175-185, 2008.

PIZAURO, J. R.; CIANCAGLINI, P.; MACARI, M. Discondroplasia tibial: mecanismo de lesao e controle; Revista Brasileira de Ciência Avícola, v.4, n.3, p.122, 2002.

RENNIE, J. S.; WHITEHEAD, C. C. The effectiveness of dietary 25- and 1-hydroxycholecalciferol in preventing tibial dyschondroplasia in broiler chickens. British Poultry Science, v.37, p.413-421, 1996.

ROSTAGNO, H. S.; ALBINO, L. F. T.; DONZELE, J. L.; GOMES, P. C.; OLIVEIRA, R. F. de; LOPES, D. 
C.; FERREIRA, A. S.; BARRETO, S. L. de T. Tabelas brasileiras para aves e suínos: composição de alimentos e exigências nutricionais. 3.ed. Viçosa: UFV, 2005.

SAS Institute. SAS Proprietary Software Release 9.2. SAS Inst. Inc., Cary, NC. 2002.

SHAFEY, T. M., McDONALD, M. W., PYM, R. A. Effects of dietary calcium, avaliable phosphorus and vitamin $\mathrm{d}$ on growth rate, food utilization, plasma and bone constituents and calcium and phosphorus retention of commercial broiler strains. British Poultry Science, v.31, n.3, p.587-602, 1990.

SILVA, D. J.; QUEIROZ, A. C. Análise de alimentos: métodos químicos e biológicos. 3.ed. Viçosa: UFV, 2002.

TORRES, C. A.; VIEIRA, S. L.; REIS, R. N.; FERREIRA, A. K.; SILVA, P. X.; FURTADO F. V. F. Productive performance of broiler breeder hens fed 25hydroxycholecalciferol. Revista Brasileira de Zootecnia, v.38, n.7, p.1286-1290, 2009.
VELTMANN, JR. J. R; JENSEN, L. S. Tibial Dyschondroplasia in Broilers: Comparison of Dietary Additives and Strains. Poultry Science, v.60, p.14731478, 1981.

WALDROUP, P. W.; STEARNS, J. E.; AMMERMAN, C. B.; HARMS, R. H. Studies on the vitamin D3 requirement of the broiler chick. Poultry Science, v.44, p.543-548, 1965.

WHITEHEAD, C.C.; MCCORMACK, H. A.; MCTIER, L.; FLEMING, R. H. High vitamin $\mathrm{D}_{3}$ requirements in broilers for bone quality and prevention of tibial dyschondroplasia and interactions with dietary calcium, available phosphorus and Vitamin A. British Poultry Science, v.45, p. 425-436, 2004.

WILLIAMS, B.; WADDINGTON, D.; SOLOMON, S.; FARQUHARSON, C. Dietary effects on bone quality and turnover, and $\mathrm{Ca}$ and $\mathrm{P}$ metabolism in chickens. Research in Veterinary Science, v.69, p.8187, 2000. 